\title{
Is a Single Conformer Sufficient to Describe the Reorganization Energy of Amorphous Organic Transport Materials?
}

\author{
J. Terence Blaskovits ${ }^{\dagger \S}$, Kun-Han Lin ${ }^{\dagger \S}$, Raimon Fabregat ${ }^{\dagger}$, Iwona Swiderska ${ }^{\dagger}$, Hélène $\mathrm{Wu}^{\dagger}$, and \\ Clémence Corminboeuf ${ }^{*}$ \\ ${ }^{\dagger}$ Laboratory for Computational Molecular Design (LCMD), Institute of Chemical Sciences and \\ Engineering (ISIC), École Polytechnique Fédérale de Lausanne (EPFL), CH-1015 Lausanne, \\ Switzerland.
}

E-mail: clemence.corminboeuf@epfl.ch

\begin{abstract}
The reorganization energy $(\lambda)$, which quantifies the structural rearrangement of a molecule when accommodating a charge, is a key parameter in the evaluation of charge mobility in molecular solids. However, it is unclear how $\lambda$ is influenced by conformational isomerism, which co-exist in amorphous solids. Here, we examine the conformational space of a family of model amorphous organic hole transport materials (HTMs), derived from triphenylamine in a core-arm template, and probe the effect of conformational complexity on $\lambda$. We observe an extreme dependence of $\lambda$ on the conformer geometry of sterically congested HTMs, which to the best of our knowledge has not been described previously. These results serve as a cautionary tale that, while extracting the reorganization energy from a single molecular conformer optimized in the gas phase may be appropriate for rigid and sterically unencumbered structures, it is not for many state-of-the-art HTMs that contain multiple bulky substituents.
\end{abstract}


Charge transport layers are crucial for the high efficiency of organic photovoltaics and light-emitting diodes. The search for new charge transport materials is a driving force behind research aiming to optimize the architecture of optoelectronic devices. ${ }^{1-3}$ Amorphous organic hole transport materials (HTMs) in particular are industrially attractive due to their low cost of fabrication, modular synthesis, solution processability and amenability to vapor deposition techniques. ${ }^{4,5}$

Charge mobility is the key property that governs the performance of a bulk HTM. In amorphous HTMs, the charge transport process can be approximated using the hopping transport picture. In the nonadiabatic limit, the rate constant $k$ for a charge hopping between two molecules is described by the Marcus rate equation ${ }^{6,7}$ :

$$
k=\frac{2 \pi}{\hbar}|V|^{2} \frac{1}{\sqrt{4 \pi \lambda k_{B} T}} \exp \left(-\frac{\left(\lambda+\Delta G^{\circ}\right)^{2}}{4 \lambda k_{B} T}\right)
$$

where $V$ is the electronic coupling between the two sites, $\lambda$ is the reorganization energy, $\Delta G^{\circ}$ is the difference between the free energy of the initial and final states (i.e. the driving force), $k_{B}$ is the Boltzmann constant, $\mathrm{T}$ is the temperature and $\hbar$ is the reduced Planck constant. There is, therefore, great interest in evaluating and finding methods to modulate the key parameters $V$ and $\lambda$ as a means to maximize charge mobility. $V$ is defined for two diabatic states of a charge transfer molecular dimer and it is strongly dependent on the relative orientation of the dimer. In computational studies, these dimers are either generated from the most stable configuration of the dimer in the gas phase, or by taking all possible molecular pairs from a simulated bulk morphology. ${ }^{8-13}$ By contrast, the reorganization energy, which expresses the change in free energy caused by structural relaxation upon the accommodation of a charge, can be computed from the neutral and cationic potential energy surfaces of a single molecule.

Given the relative ease of evaluating reorganization energies, the minimization of $\lambda$ is often used as a descriptor in the computational screening and design of materials with promising charge transport behaviour. ${ }^{10,14,15}$ There is, however, an inconsistency in the literature with regards to the method by which to evaluate $\lambda$. In some instances, many individual structures are extracted directly from an amorphous mixture generated using QM/MM methods ${ }^{10}$, although this is computationally demanding. For a reduced computational cost, neutral and charged structures may instead be optimized with constrained soft internal coordinates (e.g. dihedrals) to mimic the restrictive effect of nearby structures in the bulk environment. ${ }^{8,9}$ This often leads to very small reorganization energies, as these techniques confine the low-energy vibrational modes. And yet, these modes may be the most important for charge transfer, given that they are the energetically most available modes at room temperature. Alternatively, $\lambda$ may simply be extracted from a single conformer which has been optimized in the ground state at a density functional theory (DFT) level. ${ }^{11,16}$ Although this approach in general works well for rigid compounds, its validity for flexible and bulky structures such as hole transport materials, where many conformers may appear in an amorphous morphology, has not been verified and warrants thorough investigation. Finally, while QM/MM-simulated bulk geometries (as described above) have also been used to study the environmental effects on reorganization energy, the targeted systems have been primarily rigid, and thus the effect of the bulk on conformational variation in flexible molecules has not been investigated.
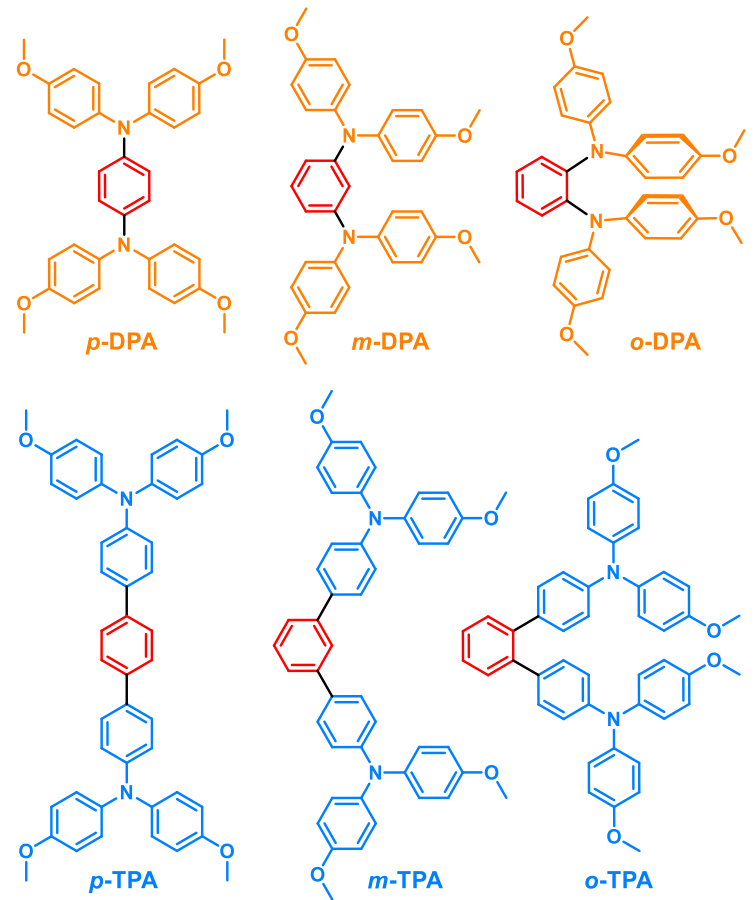

Figure 1: The six model HTMs studied in this work, containing a benzene core (red), and para-methoxy substituted arms derived from diphenylamine (DPA, orange) and triphenylamine (TPA, blue).

HTMs are often designed using a core-arm template, whereby large and orthogonally oriented arms are used 
to prevent aggregation and preclude crystallization of the transport layer. ${ }^{5}$ The design of the arms influences bulk morphology, and an increased number of arms reduces reorganization energy ${ }^{12,17}$ and improves charge mobility. ${ }^{12,18,19}$ Furthermore, the interactions involving these arms at the organic-inorganic interface of perovskites are critical components of an effective HTM. ${ }^{20,21}$ However, the presence of such sterically congested and flexible arms may lead to a complex free energy landscape due to significant intramolecular interactions, which are challenging to model, ${ }^{22,23}$ and the consequences of conformational diversity on $\lambda$ are currently unclear.

To address this, we studied a family of six model corearm HTMs composed of a benzene core and branched phenylamine arms with methoxy substituents (see Figure 1). Di- and tri-phenylamine arm moieties are ubiquitous in the high-performance HTMs incorporated in dye-sensitized and perovskite solar cells $^{5}$ and organic light-emitting diodes. ${ }^{2}$ The compounds differ from one another with respect to the arm substitution pattern on the central ring - para $(p)$, meta $(m)$ and ortho $(o)$ - and in the size of the arms: diphenylamine (DPA) and triphenylamine (TPA). These substitutions enable us to establish the effect of sterically congested environments and inter-arm interactions on reorganization energy. All of these structures $^{24-27}$ (or their synthetic precursors ${ }^{28,29}$ ) have been reported experimentally, and $p$-TPA has already been used as a HTM in perovskite solar cells. ${ }^{25}$ Furthermore, these structures are of interest in electrochemistry for their charge transfer properties, as electronic coupling both via the benzene spacer (through-bond) and between the arms (through-space) are predicted. ${ }^{26,27}$

The conformational landscape of these six compounds was established using replica exchange molecular dynamics ${ }^{30}$ (REMD) enhanced sampling at $300 \mathrm{~K}$ at the DFTB-D3BJ level. These are shown as 2D free energy maps in Figure 2 (further information is given in the computational details in the SI). The minimum and maximum distances between any two oxygens of the methoxy substituents were used as collective variables.

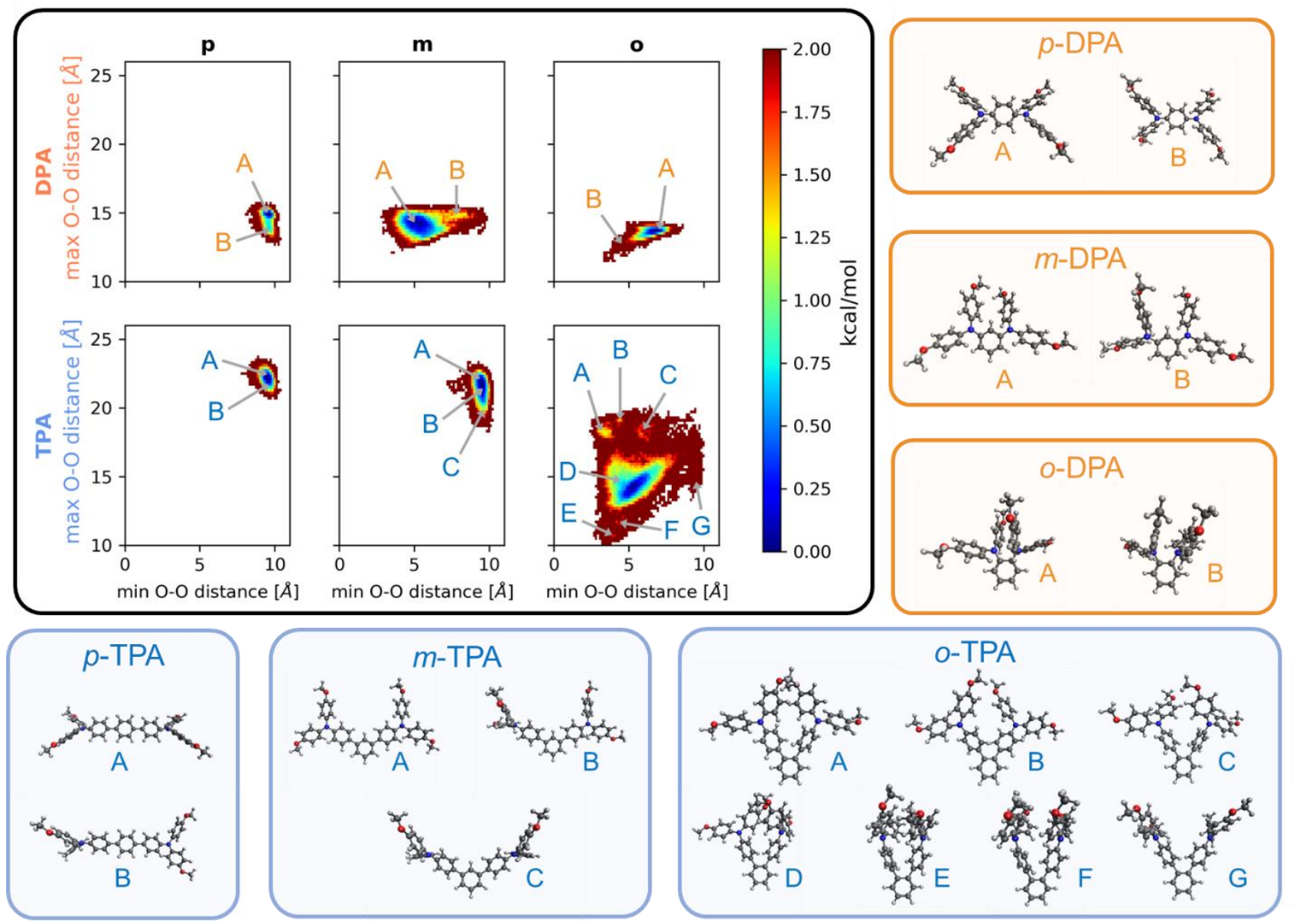

Figure 2: Free energy maps and relevant conformers for para, meta and ortho-substituted DPA (orange) and TPA (blue) structures. Labels indicate clustering of points following DFT optimization, and a representative conformer from each cluster is given. Free energy surfaces were obtained from REMD simulations at the DFTB-D3BJ level. 
We took a small sample of 500 structures equally separated in time from the thermalized replica at $300 \mathrm{~K}$ and optimized them in the gas phase using DFT at the B3LYP(D3BJ)/6-31G(d,p) level. The DFT-optimized structures migrate to well-defined clusters on the free energy plots, labelled with letters in Figure 2, which map closely to the most highly populated regions identified by REMD.

It is clear from Figure 2 that $p$-DPA, $p$-TPA and $m$-TPA exhibit very little variation in geometry, while $m$-DPA, $o$-DPA and especially $o$-TPA exhibit a more complex conformational landscape. Given that both parasubstituted compounds behave as rigid rotors, it is unsurprising that the O-O distances in $p$-DPA and $p$ TPA do not change significantly, and that the free energy difference between rotamers is negligible. In $m$ DPA and $m$-TPA, the conformers differ with respect to rotations about the amines. Depending on the rotation, the benzene rings of the $m$-DPA arms are either close enough $(\sim 4 \AA)$ to enable $\pi-\pi$ interactions $\left(m-\mathrm{DPA}_{\mathrm{A}}\right.$, where the subscript ${ }_{A}$ refers to the cluster labelled in Figure 2$)$, or are non-interacting $\left(m-\mathrm{DPA}_{\mathrm{B}}\right)$. The supplementary benzene ring in $m$-TPA increases the distance between the arms, thereby preventing interarm interactions and simplifying the conformational space.

In $o$-DPA, the proximity of the arms restricts conformational freedom: the benzene rings from each arm are either slightly disarticulated $\left(o-\mathrm{DPA}_{\mathrm{A}}\right)$, or faceto-face in a $\pi$-stacked configuration $\left(o-\mathrm{DPA}_{\mathrm{B}}\right)$. In contrast, multiple clusters $\left(o-\mathrm{TPA}_{\mathrm{A}-\mathrm{F}}\right.$ in Figure 2$)$ separated by shallow energy barriers are found in the two-dimensional free energy surface of $o$-TPA, due to the flexibility introduced by the additional benzene rings and the increased geometric possibility of interactions between the adjacent arms. These conformational clusters can be classified into those whose arms are oriented in an end-to-end fashion and have limited contact with one another via one terminal benzene ring of each arm $\left(o-\mathrm{TPA}_{\mathrm{A}-\mathrm{D}}\right)$, and those with arms which are completely co-facial to one another and exhibit significant non-covalent interactions $\left(o-\mathrm{TPA}_{\mathrm{E}-}\right.$ F). We term the latter arrangements 'self-aggregated'. Only one cluster $\left(o-\mathrm{TPA}_{\mathrm{G}}\right)$ does not show any evidence of interarm interactions, such that the minimum O-O atom distance reported is simply the distance between the two oxygen atoms on the same arm $(\sim 9.6 \AA)$.

To establish the effect of conformational complexity on the range of possible reorganization energies in these compounds, $\lambda$ was computed for the DFT-optimized conformers using the four-point method. The distribution of $\lambda$ for each HTM is shown as violin plots in Figure 3 and the regions in these plots are associated to the clusters labelled in Figure 2. The range of $\lambda$ values obtained is clearly representative of conformational complexity observed in the free energy landscape. The three 'rigid' HTMs with non-interacting arms ( $p$-DPA, $p$-TPA and $m$-TPA) exhibit the narrowest distributions of $\lambda$, while the HTMs with the geometric capacity for interarm interactions, and therefore more complex conformational landscapes $(m$ DPA and especially $o$-TPA), exhibit a broader range of reorganization energies. The overall lower average values of $\lambda$ in TPA compared to DPA is attributed to a higher delocalization of the charge across the larger conjugated system in the TPA family.
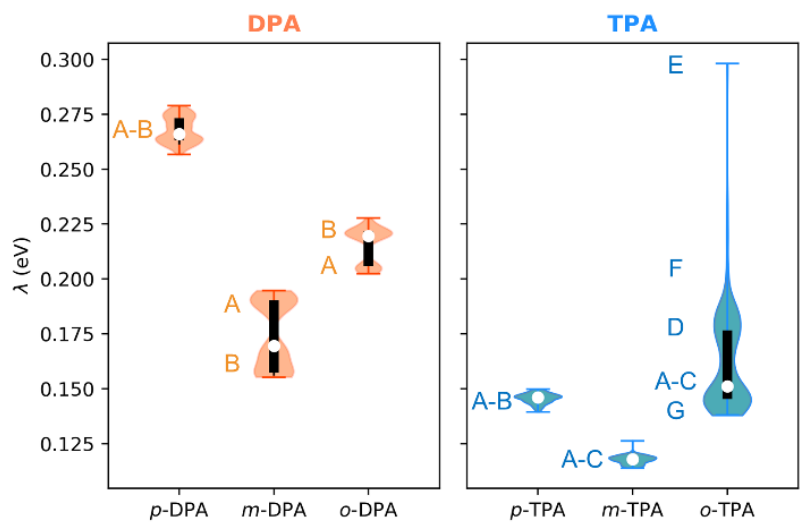

Figure 3: Violin plots of the reorganization energy probability density for 500 conformers of para, meta and ortho-substituted DPA (orange) and TPA (blue). The white point indicates the average value, the black bar the range between the $1^{\text {st }}$ and $3^{\text {rd }}$ quartiles, and the whiskers the maximal and minimal values. Letters correspond to the clusters labelled in Figure 2. Neutral and cationic geometries were optimized at the B3LYP(D3BJ)/6$31 \mathrm{G}(\mathrm{d}, \mathrm{p})$ level, and reorganization energy was computed using the 4-point method (see the SI for computational details).

The relationship between larger arm-arm interactions and increased $\lambda$ can be extended to the various conformational clusters of the individual compounds (Figure 3). For instance, in $m$-DPA and $o$-DPA, the conformer clusters with greater interarm interactions $\left(m-\mathrm{DPA}_{\mathrm{A}}\right.$ and $\left.o-\mathrm{DPA}_{\mathrm{B}}\right)$ have systematically higher $\lambda$ than the less-interacting clusters $\left(m-\mathrm{DPA}_{\mathrm{B}}\right.$ and $o$ $\mathrm{DPA}_{\mathrm{A}}$ ). However, the most striking example of this trend is found in the conformational diversity of $o$ TPA. While the non-interacting cluster $o$-TPA displays the lowest reorganization energies (as low as $0.14 \mathrm{eV}$ ), the end-to-end oriented arm clusters 

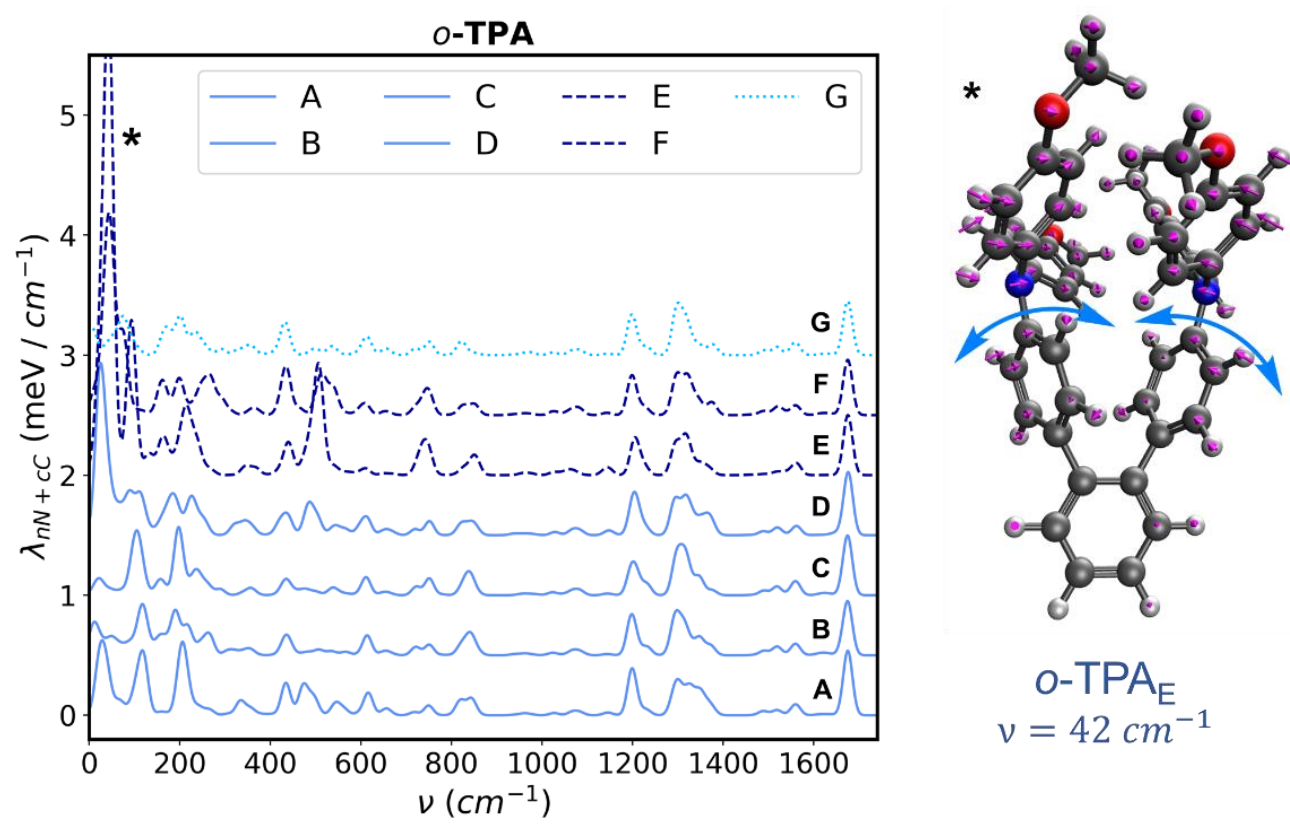

Figure 4: Decomposition of the normal mode contributions to the total reorganization energy of a representative structure from each conformational cluster of $o$-TPA, coloured by the interacting (solid lines, $o$-TPA $\mathrm{T}_{\mathrm{A}-\mathrm{D}}$ ), self-aggregated (dashed lines, $o$-TPA $\mathrm{E}_{\mathrm{F}}$ ) and non-interacting (dotted line, $o$-TPA $A_{\mathrm{G}}$ geometries of the arms. The vibrational mode corresponding to the highest peak in the self-aggregated cluster (marked with $*$ ) is shown to the right with the small purple arrows, and the overall motion is depicted with the large blue arrows.

$o-\mathrm{TPA}_{\mathrm{A}-\mathrm{C}}$ and the more interacting cluster $o-\mathrm{TPA}$ are somewhat higher, and the self-aggregated clusters $o$ $\mathrm{TPA}_{\mathrm{E}}$ and $o-\mathrm{TPA}_{\mathrm{F}}$ are significantly larger than the rest of the distribution, with $\lambda$ values up to $0.30 \mathrm{eV}$. As with $m$-DPA and $o$-DPA, it is clear that conformers with more substantial interarm interactions contribute to notably higher reorganization energies than conformers without such interactions.

Normal mode analysis (NMA) was performed on a representative system from each compound cluster to establish the contributions of the individual vibrational modes to the total molecular reorganization energy (see the SI for computational details). The variation in $\lambda$, both between conformational clusters of the same HTM and across the six different HTMs, lies primarily in soft modes below $200 \mathrm{~cm}^{-1}$ (see Figure S1 for DPA and Figure 4 and Figure S2 for TPA). In $o$-TPA, the softmode contributions and overall $\lambda$ of the various clusters correlate well with one another (see Figure S3) and with the degree of interaction between the arms seen in Figure 2, according to the general trend $o-\mathrm{TPA}_{\mathrm{G}}<o$ $\mathrm{TPA}_{\mathrm{A}-\mathrm{C}}<o-\mathrm{TPA}_{\mathrm{D}}<o-\mathrm{TPA}_{\mathrm{F}}<o-\mathrm{TPA}_{\mathrm{E}}$. In $o-\mathrm{TPA}_{\mathrm{E}}$ and $o-\mathrm{TPA}_{\mathrm{F}}$, the conformers with the greatest contributions to $\lambda$ below $200 \mathrm{~cm}^{-1}$, the most significant normal mode is a breathing-like mode involving gliding and repulsion motions between the adjacent arms (see
Figure 4 for this mode in $o-\mathrm{TPA}$ ). The next cluster with the most substantial inter-arm interactions, $o$ $\mathrm{TPA}_{\mathrm{D}}$, also exhibits a similar peak, albeit smaller than those in the self-aggregated clusters, while the soft modes in $o-\mathrm{TPA}_{\mathrm{A}-\mathrm{C}}$ and the non-interacting cluster $o$ $\mathrm{TPA}_{\mathrm{G}}$ contribute very little to the total reorganization energy.

Likewise, in ortho-substituted DPA the higher $\lambda$ observed in the cluster with the most heavily interacting arms $\left(o-\mathrm{DPA}_{\mathrm{B}}\right)$ is attributed to the presence of large normal-mode contributions in the same region, as compared to the less-interacting disarticulated arms in $o$-DPA (see Figure S1). Furthermore, in both the DPA and TPA families, the meta-substituted isomer consistently displays the lowest $\lambda$, which correlates with reduced soft mode contributions (see Figure 3 and Figures S1-S2). Clearly, the dependence of $\lambda$ on conformer geometry originates from these interfragment modes, and the range of $\lambda$ can be traced to the varying degree of interaction between an HTM's arm fragments. The observation that a broad range of possible reorganization energies is accessible to $o$-TPA raises the question of if the high $\lambda$ observed in the gasphase self-aggregated conformers is in any way representative of the $\lambda$ of an amorphous mixture, or if $\lambda$ 
is strongly altered by structural changes induced by the bulk environment.

To answer this, we simulated the behavior of a selfaggregated structure in the solid state. Obtaining a conformational diversity which is representative of an amorphous solid-state material is often done using force fields in a 'melt and quench' procedure, which preserves the randomly oriented nature of structures obtained at a higher temperature. To assess if a force field is an appropriate method by which to establish the conformational space and thus to generate a representative bulk morphology of the HTMs studied here, a REMD simulation using a classical force field was tested but was found to be unsuitable (see Figure S4). We therefore turned to a two-part QM/MM mechanical embedding simulation strategy to evaluate the effect of the bulk environment on the $o-\mathrm{TPA}$ cluster. To generate the amorphous morphology, we embedded a self-aggregated structure from the $o-\mathrm{TPA}$ cluster with 100 other $o$-TPA $\mathrm{D}_{\mathrm{D}}$ molecules and performed an MD simulation, during which the selfaggregated structure was kept frozen to avoid any conformational transformation. This central conformer and surrounding layer of structures were then extracted and relaxed using a subsequent QM/MM optimization (see the SI for computational details). While the central structure remains in the self-aggregated conformation, the $\lambda$, which is evaluated at $0.296 \mathrm{eV}$ for that particular conformer in the gas phase, falls to $0.200 \mathrm{eV}$ in the bulk. This significant difference is attributed to the change in this HTM's capacity to reorient its arms upon accommodation of the charge. To quantify this, we collected the difference in distance between the nitrogen atoms of the adjacent arms in the neutral and cationic geometries $\left(\Delta d_{N-N}\right)$. While $\Delta d_{N-N}$ is large for the self-aggregated structure in the gas phase $(0.54 \AA)$, it decreases substantially in the condensed phase $(0.21$ $\AA$, see Figure S5 for a summary of the change in armarm distances and $\lambda$ in the gas and condensed phases), showing that the contributions of strong interarm interactions which lead to high $\lambda$ in the gas phase are lost in the bulk environment.

The same two-step QM/MM protocol was applied to a structure from the most abundant cluster $o-\mathrm{TPA}_{\mathrm{D}}(\lambda=$ $0.141 \mathrm{eV}$ in the gas phase). The neutral and cationic geometries obtained in the simulated bulk lead to a slight increase of $\lambda$ to $0.168 \mathrm{eV}$. Similarly, $\Delta d_{N-N}$ increases from $0.06 \AA$ in the gas phase to $0.20 \AA$ (see Figure S5). These results suggest that the degree of geometric relaxation permitted in the bulk is similar for both the self-aggregated and less-interacting structures, due to the restrictive effect of the adjacent molecules. This leads to overall much more similar values of $\lambda$ for two different conformers than what is suggested by the conformer-dependent distribution obtained in the gasphase (see Figure 3).

In conclusion, we have demonstrated a strong dependence of reorganization energy on conformer geometry in a series of model core-arm HTMs derived from diphenylamine and triphenylamine. Replicaexchange molecular dynamics simulations reveal that the conformational space of rigid and sterically unhindered compounds ( $p$-DPA, $p$-TPA and $m$-TPA) is uncomplicated, giving rise to a narrow distribution of computed reorganization energies. To the contrary, flexible compounds with many degrees of freedom (oDPA, $m$-DPA and especially $o$-TPA) exhibit a wide range of $\lambda$ values, spanning up to $0.150 \mathrm{eV}$ in the case of $o$-TPA. Substantial intramolecular interactions between bulky arms stabilize self-aggregated geometries in the gas phase and lead to the emergence of arm-arm 'breathing'-like vibrational modes. These features contribute to surprisingly high $\lambda$ values which, when compared to results from $\mathrm{QM} / \mathrm{MM}$ simulations on these same geometries, are not found to be representative of those obtained in a bulk environment.

It is important to highlight that such interactions are found widely in many solution-processible amorphous charge transport materials. Thus, these compounds are precisely among those which are most at risk for a severe overestimation of reorganization energy, as demonstrated here. In the context of screening materials for low reorganization energy, the approach of computing $\lambda$ from a lowest energy conformer in the gas phase (e.g. following a conformation search) is therefore not appropriate for flexible structures, as it may severely overestimate $\lambda$. We stress that results obtained from a simulation of the bulk are preferred, due to the more realistic description of the effect of the surrounding environment on the molecule's structural response to charge accommodation. Furthermore, we have shown that a force field which has not been parametrized for non-covalent interactions does not appropriately describe the conformational space of such problematic compounds. We advise that care must be taken in choosing the method for simulating the conformational diversity and resulting properties of flexible charge transport materials. 


\section{SUPPORTING INFORMATION}

Details of all computational procedures, normal mode analysis of representative structures, REMD(GAFF) free energy map, and results of QM/MM simulations.

\section{AUTHOR INFORMATION}

\section{Corresponding author}

E-mail: clemence.corminboeuf@epfl.ch

\section{Orcid}

J. Terence Blaskovits: 0000-0002-1452-5508

Kun-Han Lin: 0000-0001-6689-667X

Clémence Corminboeuf: 0000-0001-7993-2879

\section{Author Contributions}

$\S$ J. T. B. and K.-H. L. contributed equally.

\section{Notes}

The authors declare no competing financial interest.

\section{ACKNOWLEDGMENT}

The authors are grateful to the EPFL for financial support and the allocation of computational resources. C.C. and R.F. acknowledge the European Research Council (ERC, Grant Agreement No. 817977).

\section{REFERENCES}

(1) Kim, G.-W.; Choi, H.; Kim, M.; Lee, J.; Son, S. Y.; Park, T. Hole Transport Materials in Conventional Structural (n-i-p) Perovskite Solar Cells: From Past to the Future. Adv. Energy Mater. 2020, 10, 1903403.

(2) Shahnawaz; Sudheendran Swayamprabha, S.; Nagar, M. R.; Yadav, R. A. K.; Gull, S.; Dubey, D. K.; Jou, J.-H. Hole-transporting materials for organic light-emitting diodes: an overview. $J$. Mater. Chem. C 2019, 7, 7144-7158.

(3) Kulkarni, A. P.; Tonzola, C. J.; Babel, A.; Jenekhe, S. A. Electron Transport Materials for Organic Light-Emitting Diodes. Chem. Mater. 2004, 16, 4556-4573.

(4) Allard, S.; Forster, M.; Souharce, B.; Thiem, H.; Scherf, U. Organic Semiconductors for Solution-Processable Field-Effect Transistors (OFETs). Angew. Chem. Int. Ed. 2008, 47, 40704098.
(5) Agarwala, P.; Kabra, D. A review on triphenylamine (TPA) based organic hole transport materials (HTMs) for dye sensitized solar cells (DSSCs) and perovskite solar cells (PSCs): evolution and molecular engineering. J. Mater. Chem. A 2017, 5, 1348-1373.

(6) Marcus, R. A.; Sutin, N. Electron transfers in chemistry and biology. Biochim. Biophys. Acta, Rev. Bioenerg. 1985, 811, 265-322.

(7) Marcus, R. A. Chemical and electrochemical electron-transfer theory. Annu. Rev. Phys. Chem. 1964, 15, 155-196.

(8) Friederich, P.; Meded, V.; Poschlad, A.; Neumann, T.; Rodin, V.; Stehr, V.; Symalla, F.; Danilov, D.; Lüdemann, G.; Fink, R. F.; Kondov, I.; von Wrochem, F.; Wenzel, W. Molecular Origin of the Charge Carrier Mobility in Small Molecule Organic Semiconductors. Adv. Funct. Mater. 2016, 26, 5757-5763.

(9) Rühle, V.; Lukyanov, A.; May, F.; Schrader, M.; Vehoff, T.; Kirkpatrick, J.; Baumeier, B.; Andrienko, D. Microscopic Simulations of Charge Transport in Disordered Organic Semiconductors. J. Chem. Theory Comput. 2011, 7, 3335-3345.

(10) Schober, C.; Reuter, K.; Oberhofer, H. Virtual Screening for High Carrier Mobility in Organic Semiconductors. J. Phys. Chem. Lett. 2016, 7, 3973-3977.

(11) Yavuz, I.; Lin, J. B.; Houk, K. N. Impact of morphology, side-chains, and crystallinity on charge-transport properties of $\pi$-extended double helicenes. Phys. Chem. Chem. Phys. 2019, 21, 901914.

(12) Lin, K.-H.; Prlj, A.; Yao, L.; Drigo, N.; Cho, H.-H.; Nazeeruddin, M. K.; Sivula, K.; Corminboeuf, C. Multiarm and Substituent Effects on Charge Transport of Organic Hole Transport Materials. Chem. Mater. 2019, 31, 6605-6614.

(13) Lin, K.-H.; Prlj, A.; Corminboeuf, C. How does alkyl chain length modify the properties of triphenylamine-based hole transport materials? $J$. Mater. Chem. C 2018, 6, 960-965.

(14) Kunkel, C.; Schober, C.; Margraf, J. T.; Reuter, K.; Oberhofer, H. Finding the Right Bricks for Molecular Legos: A Data Mining Approach to Organic Semiconductor Design. Chem. Mater. 2019, 31, 969-978. 
(15) Gryn'ova, G.; Lin, K.-H.; Corminboeuf, C. Read between the Molecules: Computational Insights into Organic Semiconductors. J. Am. Chem. Soc. 2018, 140, 16370-16386.

(16) Deng, J.; Hu, W.; Shen, W.; Li, M.; He, R. Exploring the electrochemical properties of hole transporting materials from first-principles calculations: an efficient strategy to improve the performance of perovskite solar cells. Phys. Chem. Chem. Phys. 2019, 21, 1235-1241.

(17) Lin, K.-H.; Corminboeuf, C. FB-REDA: fragment-based decomposition analysis of the reorganization energy for organic semiconductors. Phys. Chem. Chem. Phys. 2020, 22, 11881-11890.

(18) Park, S.; Heo, J. H.; Yun, J. H.; Jung, T. S.; Kwak, K.; Ko, M. J.; Cheon, C. H.; Kim, J. Y.; Im, S. H.; Son, H. J. Effect of multi-armed triphenylamine-based hole transporting materials for high performance perovskite solar cells. Chem. Sci. 2016, 7, 5517-5522.

(19) Liu, X.; Kong, F.; Jin, S.; Chen, W.; Yu, T.; Hayat, T.; Alsaedi, A.; Wang, H.; Tan, Z. a.; Chen, J.; Dai, S. Molecular Engineering of Simple Benzene-Arylamine Hole-Transporting Materials for Perovskite Solar Cells. ACS Appl. Mater. Interfaces 2017, 9, 27657-27663.

(20) Torres, A.; Rego, L. G. C. Surface Effects and Adsorption of Methoxy Anchors on Hybrid Lead Iodide Perovskites: Insights for SpiroMeOTAD Attachment. J. Phys. Chem. C 2014, 118, 26947-26954.

(21) Yin, J.; Cortecchia, D.; Krishna, A.; Chen, S.; Mathews, N.; Grimsdale, A. C.; Soci, C. Interfacial Charge Transfer Anisotropy in Polycrystalline Lead Iodide Perovskite Films. $J$. Phys. Chem. Lett. 2015, 6, 1396-1402.

(22) Petraglia, R.; Nicolaï, A.; Wodrich, M. D.; Ceriotti, M.; Corminboeuf, C. Beyond static structures: Putting forth REMD as a tool to solve problems in computational organic chemistry. $J$. Comput. Chem. 2016, 37, 83-92.

(23) Fabregat, R.; Fabrizio, A.; Meyer, B.; Hollas, D.; Corminboeuf, C. Hamiltonian-
Reservoir Replica Exchange and Machine Learning Potentials for Computational Organic Chemistry. J. Chem. Theory Comput. 2020, 16, 3084-3094.

(24) Uebe, M.; Kazama, T.; Kurata, R.; Sakamaki, D.; Ito, A. Recognizing Through-Bond and Through-Space Self-Exchange Charge/Spin Transfer Pathways in Bis(triarylamine) Radical Cations with Similar Geometrical Arrangements. Angew. Chem. Int. Ed. 2017, 56, 15712-15717.

(25) Wu, J.; Liu, C.; Deng, X.; Zhang, L.; Hu, M.; Tang, J.; Tan, W.; Tian, Y.; Xu, B. Simple and low-cost thiophene and benzene-conjugated triaryamines as hole-transporting materials for perovskite solar cells. RSC Adv. 2017, 7, 4547845483.

(26) Coropceanu, V.; Gruhn, N. E.; Barlow, S.; Lambert, C.; Durivage, J. C.; Bill, T. G.; Nöll, G.; Marder, S. R.; Brédas, J.-L. Electronic Couplings in Organic Mixed-Valence Compounds: The Contribution of Photoelectron Spectroscopy. J. Am. Chem. Soc. 2004, 126, 2727-2731.

(27) Nöll, G.; Avola, M. Optically induced electron transfer in an $\mathrm{N}, \mathrm{N}, \mathrm{N}^{\prime}, \mathrm{N}^{\prime}$-tetraanisyl-ophenylenediamine radical cation. J. Phys. Org. Chem. 2006, 19, 238-241.

(28) Ito, A.; Uebe, M.; Kurata, R.; Yano, S.; Fueno, H.; Matsumoto, T.

Diazadibora[1.1.1.1]m,p,m,p-cyclophanes: Ambipolar Conjugated Macrocycles with Different $\mathrm{B}-\pi-\mathrm{N}$ Embedded Patterns. Chem. Asian J. 2018, $13,754-760$.

(29) Córsico, E. F.; Rossi, R. A. Sequential Photostimulated Reactions of Trimethylstannyl Anions with Aromatic Compounds Followed by Palladium-Catalyzed Cross-Coupling Processes. $J$. Org. Chem. 2002, 67, 3311-3316.

(30) Sugita, Y.; Okamoto, Y. Replica-exchange molecular dynamics method for protein folding. Chem. Phys. Lett. 1999, 314, 141-151. 


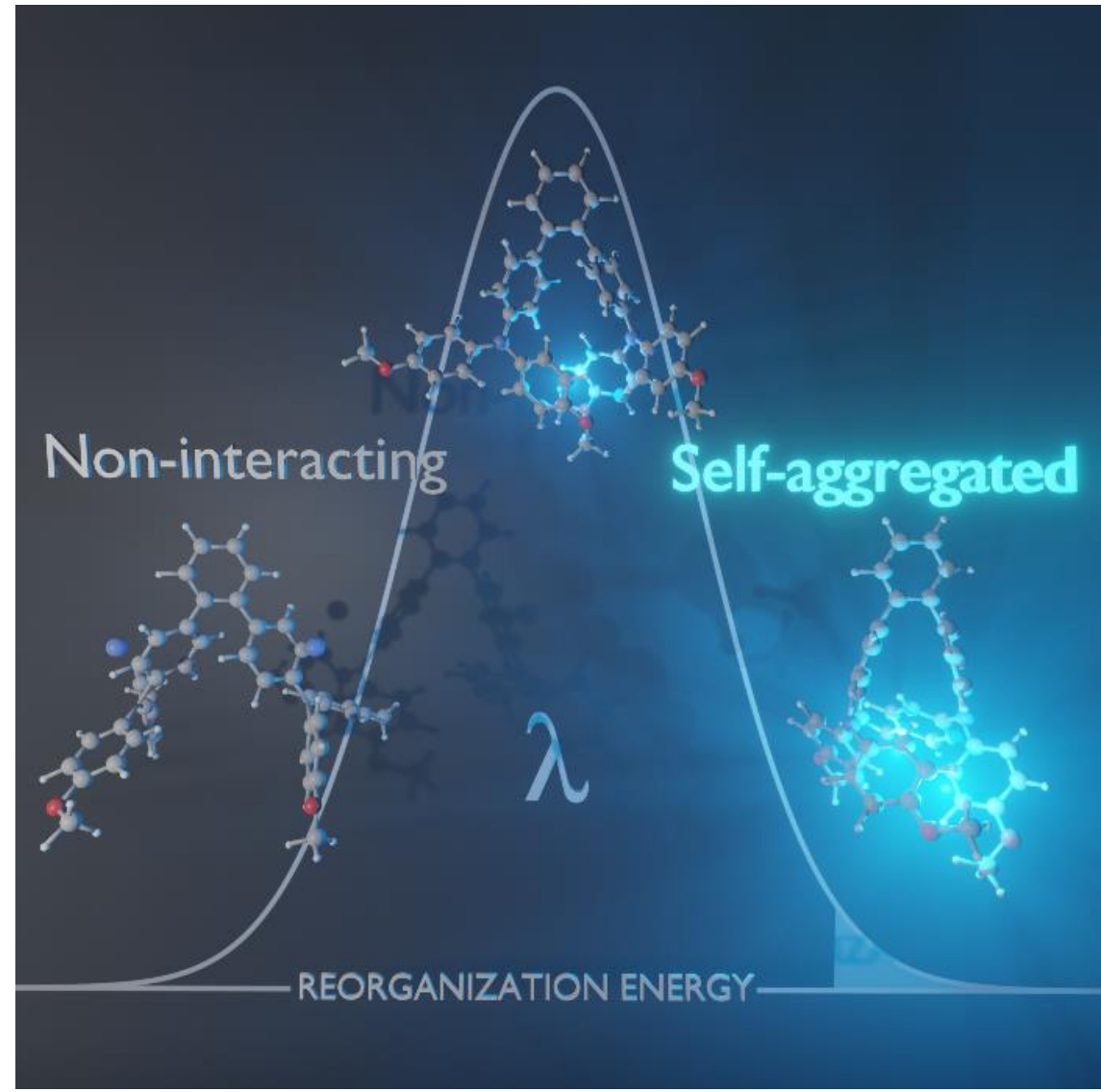

